\title{
An Innovative Solution Based on Virtual Reality to Treat Phobia
}

\author{
Iulia-Cristina Stănică ${ }^{1}$, Maria-Iuliana Dascălu ${ }^{2}$, Alin Moldoveanu ${ }^{3}$ and Florica \\ Moldoveanu $^{4}$ \\ ${ }^{1,2,4}$ Department of Engineering in Foreign Languages, University POLITEHNICA of Bucharest, Splaiul \\ Independentei, Bucharest, Romania \\ ${ }^{3}$ Faculty of Automatic Control and Computer Science, University POLITEHNICA of Bucharest, Splaiul \\ Independentei, Bucharest, Romania
}

Correspondence should be addressed to: Iulia-Cristina Stănică; iulia.stanica@gmail.com

Received date: 5 September 2016; Accepted date: 26 December 2016;

Published date: 21 February 2017

Academic Editor: Raja Akermi

Copyright (C) 2017. Iulia-Cristina Stănică, Maria-Iuliana Dascălu, Alin Moldoveanu and Florica Moldoveanu . Distributed under Creative Commons CC-BY 4.0

\begin{abstract}
The current paper conducts research on the existing methods for treating phobias, focusing on virtual reality as an innovative treatment method. After conducting a survey of the current applications using this type of treatment, we have developed our own software, Anti-PHOBIES, which proposes new ideas for treating two common phobias: claustrophobia and acrophobia. The application has been tested on healthy and suffering people, with impressive results. The main purpose of the project was to use the advantages provided by an emerging technology, virtual reality, in order to modernize the medical field and help the suffering people overcome their immense fears: phobias.
\end{abstract}

Keywords: phobias, emerging technology, virtual reality, treatment.

\section{Introduction}

Nowadays, we observe an alarming increase in the number of people affected by mental disorders. These statistics are even more worrying if we take into account the fact that this type of conditions is really difficult to be treated. Anxiety disorders are a frequent type of mental disorders, where the sufferer shows feelings of fear and anxiety. Fear appears as an immediate response to present events, while anxiety is a feeling representing discomfort, uneasiness of the mind and uncertainty caused by future events (Arlington 2003). There are various types of anxiety disorders, some important examples being phobias, panic disorder, social anxiety disorder or generalized anxiety disorder. As Wittchen \& all mention in their research paper, anxiety disorders are way more frequent than other mental disorders (Fig. 


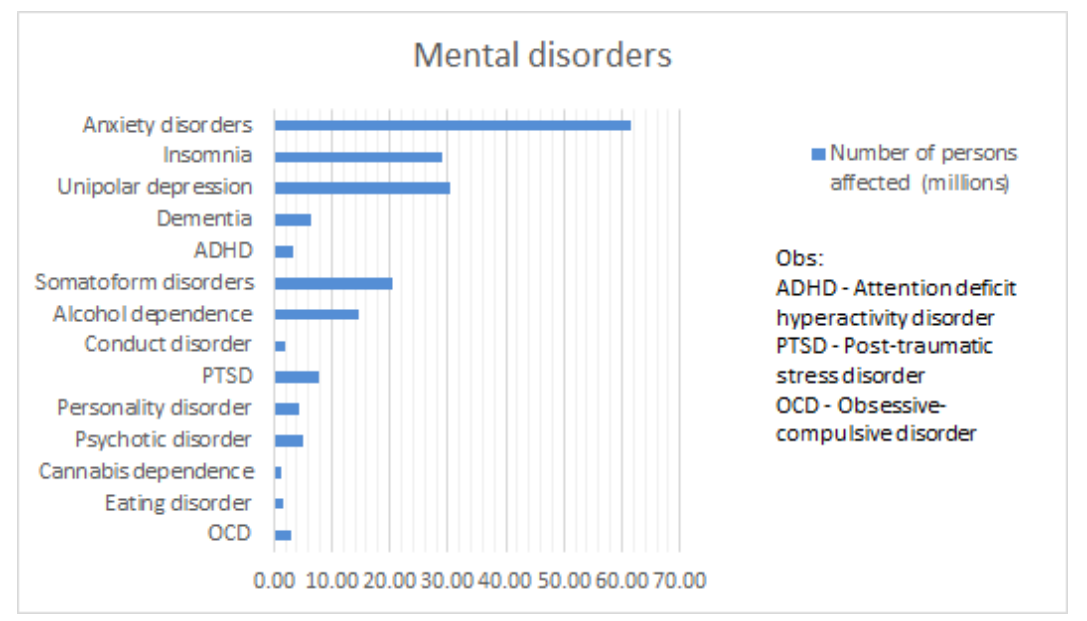

Figure 1: Repartition of mental disorders in Europe

Unfortunately, even if statistics regarding mental disorders are worrying, the sufferers can be tempted to avoid treatment, because several methods are expensive and inaccessible. The report made by Wittchen \& all shows that in 2011, $38.2 \%$ of the population of Europe were suffering of at least one mental disorder, considerably higher than the previous $27.4 \%$ from their 2005 analysis. The number of suffering people is growing continuously and this fact has a major impact upon the global economy. It is estimated that the global costs for treating mental disorders have reached the colossal sum of 1.85 billion euros, much more than the costs for treating cancer, diabetes, cardiovascular diseases or respiratory conditions (Insel 2011).

Statistics show that phobias are the most common type of anxiety disorders and that they affect a huge number of people around the world (Alonso \& all, 2004). Each person suffers at least one time in their lifetime of a non-justified fear. When this fear attains a high intensity, it means that the person suffers from a phobia. Modern medicine is constantly evolving, but a universal treatment for phobias is yet to be found. There are hundreds of well-known phobias, such as arachnophobia (fear of spiders), agoraphobia (fear of open spaces), acrophobia (fear of heights) or claustrophobia (fear of enclosed spaces), as well as bizarre phobias, for instance coulrophobia (fear of clowns). In certain situations, specialists think that the psychical condition can be treated by simply avoiding the phenomenon which causes fear; in other cases, the exact contrary: phobias can be cured by gradually exposing the patient to the object of fear (Arlington 2013).

In recent years, specialists have started seeking other psychotherapeutic methods, innovative ones, in order to help people surpass their immense fears and therefore improve their quality of life. Virtual reality (VR) is a popular current, a technology that creates a highly realistic virtual environment which could have many applications in daily life: flight simulation (Aslandere \& all), surgery training (Moglia \& all), even dance training (Chan \& all).

Our paper has the goal of presenting AntiPHOBIES, an innovative application for treating phobias. Our application is based on an emerging technology, virtual reality, and has the goal of reducing the costs needed for treating this type of mental disorders. Anti-PHOBIES has been implemented after surveying similar 
applications and it tries to improve the quality of life for the phobia sufferers.

\section{Types of treatment for phobias}

Phobias can be described as immense fears towards situations or objects that are hardly dangerous or even completely harmless. In Arlington, 2003, phobia is described as a "marked and persistent fear that is excessive or unreasonable, cued by the presence or anticipation of a specific object or situation". The patient behaves irrationally as a response to the object causing the phobia, by doing everything he can in order to evade it (Arlington 2003). Phobias manifest through some specific symptoms, physical (heavy breathing, dizziness, trembling, heart pounding or sweating) and emotional (panic, anxiety, incapacity of controlling one's own fear, despite being conscious of overreacting, fainting sensation, need to escape from a place or situation) (Smith, Segal \& Segal 2016).

In general, most anxiety disorders have a low probability of remission, after being treated. It is also the case of phobias. Today, we can find a certain number of alternative treatment methods for these conditions: some of them include being exposed gradually to the object of fear, while others suppose the complete avoidance of it, if possible.

It is a well-known fact that a low percentage of phobia sufferers seek treatment for their conditions. This behavior can be explained by some causes: many people believe there is no actual treatment for phobias or that the existing methods of treatment are inefficient. Others are scared by the idea of confronting the object of fear, giving the fact that a possible treatment could involve gradual exposure. There are also various treatment methods which are expensive for most patients or lack accessibility (Taylor, Horowitz, Powers \& Telch 2008; Matthews, Naran \& Kirkby 2016). In the following subsections, we will present some of the most used methods for treating phobias.

\section{Traditional Treatment}

\section{Medical treatment}

Some people consider that medicaments can be used to treat any kind of conditions, psychical or physical. However, many mental disorders, including phobias, do not have a universal pill which could cure any person, suffering of any phobia, in any type of situation. Medical treatment consists of sedatives and antidepressants that have the goal of diminishing anxiety and therefore helping people relax. Unfortunately, they have to be used with caution, as they can cause addiction (Soeter and Kindt 2016).

\section{Cognitive Behavioral Therapy}

The most spread method of treatment is called cognitive behavioral therapy (CBT) and it is really efficient in many situations. Its main purpose is to make the patient perceive differently the object or the situation causing fear. Consequently, patients should change their thoughts and behavior towards the influence of the phobia upon their lives (Soeter and Kindt 2016). As its name suggests, CBT has two components: cognitive therapy, which describes how fear is influenced by one's pessimistic thoughts and behavioral therapy, which studies how the sufferer reacts when facing a situation causing anxiety (Counseling Directory 2016).

"In vivo" exposure therapy

This type of therapy consists in gradually exposing the sufferer to the cause of fear, without any real danger. It can be seen initially as the worst fear of the patient, because it involves the actual confrontation of the real object causing anxiety. The patient is carefully surveilled and guided by the therapist during the entire maneuver. The intensity of the exposure to the stimuli is usually increased gradually until the sufferer starts feeling more comfortable, less scared in the presence of the object of fear (Joseph \& Gray 2011). 


\section{Emerging Technologies Treatment}

As we already mentioned, the suffering people may be tempted to avoid seeking treatment because certain methods are expensive and inaccessible. It is not the case of treatment methods based on advanced information technology. These methods have a huge potential, as they could be very efficient and accessible for a great number of individuals. Some of these methods include self-exposure based on instruction, symbolic exposure (the patient sees images and videos) or virtual reality (Wolitzky-Taylor \& all 2008).

\section{Virtual Reality Treatment}

Virtual reality represents a computergenerated environment, a simulated reality through software means. The realism of a virtual reality world is very high, making users believe they are part of a real universe. The technology stimulates two senses of the user, in order to make them live the VR experience: sight and hearing (Gamberini, Chittaro, Spagnolli \& Carlesso 2015). Virtual reality treatment can be compared to the "in vivo" treatment, as it uses similar aspects. It has however the huge advantage of providing a large assortment of environments and stimuli, causing different mechanisms and psychological reactions. It is not the only advantage, as virtual reality treatment can reduce the duration of the therapy, can provide great accessibility and flexibility. In addition, patients can feel safer and be less skeptical when they have to interact with a virtual world, as opposed to confronting the object of fear in real life (Turner and Casey, 2014). Several studies have been conducted about the matter and the results are encouraging.

VR-based interventions have been extremely successful for many anxiety disorders, because this technology stimulates in a very precise manner the frightening situations and provokes natural reactions from its users (RammirezMoreno 2012).

\section{State Of The Art}

In order to establish the characteristics of our application, we have studied several similar software products that use virtual reality to treat phobias. They are applications that could be considered as important learning tools for sufferers they do not focus only on treatment, but also on the way the patients can fight against their problem (Dascalu, Moldoveanu \& Shudayfat 2014). It was an essential step before starting the conception of our application, because Anti-PHOBIES is a software guided by people's needs, not by technologies.

Spider-World is one of the first applications of this field, having the purpose of treating arachnophobia. Their first test subject was Madame M., a woman whose phobia had reached immense proportions - she was using duct tape to keep her windows and doors closed and avoid the access of spiders in her home (Hoffman 2014). The therapy was really successful - after the treatment sessions, Madame $M$. was capable of holding a real tarantula in her hand with little anxiety, proof that her phobia was treated (Hoffman \& all 2003).

PHOBOS - Phobia and Anxiety Management is a complex application which uses virtual reality to treat many phobias, such as acrophobia, arachnophobia, agoraphobia or claustrophobia (Psychological Technologies 2014). The application is really promising - it offers a large, open environment, which can be freely explored by users. In addition, there is a free demo, available for public use.

Many universities all over the world show great interest towards the development of this type of applications. The universities of Tasmania, Valencia or Delft are just a few examples and the clinical trials of their applications have had positive results (Stanica \& all 2016).

Ongoing research studies the use of VR for treating other conditions, such as posttraumatic stress, hallucinations, eating disorders, addictions or visual 
impairments. Other tests have been conducted to create distractions for burn victims, because being part of a virtual environment can diminish the pain (Coelho 2009).

\section{The Anti-PHOBIES application}

Anti-PHOBIES is an innovative application, which uses virtual reality to treat phobias. It is a software product that develops a flexible and interactive environment, oriented towards self-treatment. All the sufferers which hesitate to go to the doctor have the opportunity to experiment this innovative aspect of the application. AntiPHOBIES is focused on treating two frequent phobias: claustrophobia and acrophobia. These two phobias have different scenarios, exposed in complex environments, created with the help of virtual reality. The users have to complete several tasks in order to advance, like in a video game. The independent modules of the application allow us to add more phobias in the future, depending on the user's needs. A self-evaluation module is also present, in order to facilitate the monitoring of the patient's own progress.

After analyzing the existing applications and especially their drawbacks, we managed to determine what we want to achieve through our project. The goals of Anti-PHOBIES were the following:

- Choosing several phobias, the most frequent ones, and treating them in separate modules and scenarios; we saw that a single scenario could reduce the application's performance;

- Increasing the utility, by conceiving the application oriented towards selftreatment - it is an innovative aspect, which offers a great accessibility to the public and can help a lot of suffering people;

- Assuring a high level of the sense of presence, for a good performance - the user will be instructed to stay calm and stop the utilization of the application in case of experiencing sickness. In addition, we encourage the idea of using adjacent devices, such as fitness bracelets, which send specific sounds when the pulse attains dangerous values and the intervention must stop;

- Deciding about the technologies used for the development, analyzing the existent options and making a choice; for a better accessibility, the application is free and works really well on a medium PC, as an executable file, whole image quality can be adapted based on the computer's performances.

By taking into consideration all the aspects mentioned above, we have established a series of functional and non-functional requirements of our application. We have also analyzed the technological options concerning the game engines and the existing 3D modeling tools, in order to make the best suitable choices.

\section{Functionalities}

After establishing the wanted requirements and before starting the actual implementation of the application, we had to elaborate the scenes and the tasks for each one of the phobias: acrophobia and claustrophobia. The scenes have to take into consideration the needed elements that could awake certain stimuli needed for treating the corresponding phobia. We have got the advice of doctors concerning the chosen scenarios in order to see if they are appropriate or not. In addition, we also have to assure a certain gradual increase in the difficulty of the given tasks. A hard task given at the beginning of the scenario can scare or provoke unpleasant reactions of the patient. The users must accustom to this new world before starting the real work towards their healing. After completing the tasks of a scenario, the user receives a self-evaluation questionnaire, which is later sent by email to the doctor and to the developer for further analysis.

\section{Scenario of use}

After configuring the controls and the graphical parameters, the application starts and shows the main menu on the screen (Fig.

2).

Iulia-Cristina Stănică, Maria-Iuliana Dascălu, Alin Moldoveanu and Florica Moldoveanu (2017), International Journal of Interactive Worlds, DOI:10.5171/2017. 155350 


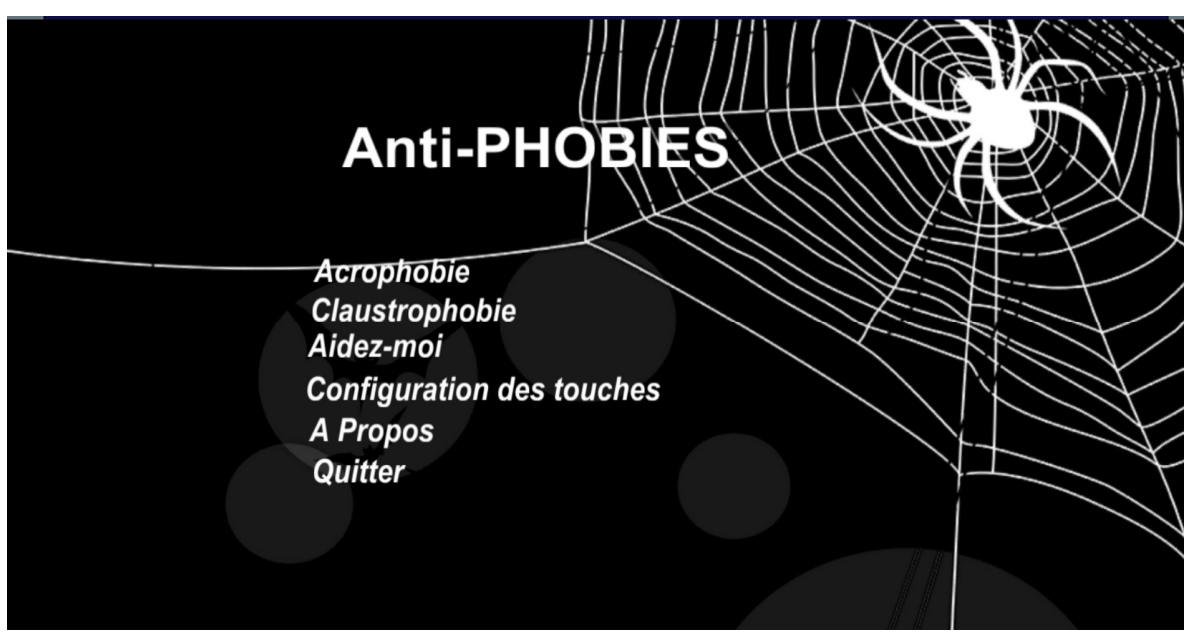

Figure 2: Main menu of Anti-PHOBIES

The menu has several options: choosing the wanted phobia, i.e. acrophobia and claustrophobia, help, to know what the goal of the application is, how the user is advised to use it and what symptoms can appear if the intensity of the stimuli is too high, key configuration, to know the predefined controls for navigation, about, which contains information related to the application, its developers and the release date and quit, to close the application.

Afterwards, the user can choose the wanted scenario based on his phobia acrophobia or claustrophobia. The two scenarios will generate specific worlds, with tasks in a fixed order, characterized by increasing levels of difficulty. For each scenario, at any time, the patient can press the "Help" button in case of emergency and the rendering of the virtual environment will be forcedly stopped.

\section{Acrophobia}

For the acrophobia, the scenario consists in a trip to the mountains (Fig. 3), where the user has the main goal of reaching the cabin situated at the top. The tasks become more and more difficult: at the beginning, the user should get used to the environment, taking a walk in the forest, admiring the view. Afterwards, he has to follow the signs, by traversing steep peaks, slopes or descending towards the abyss

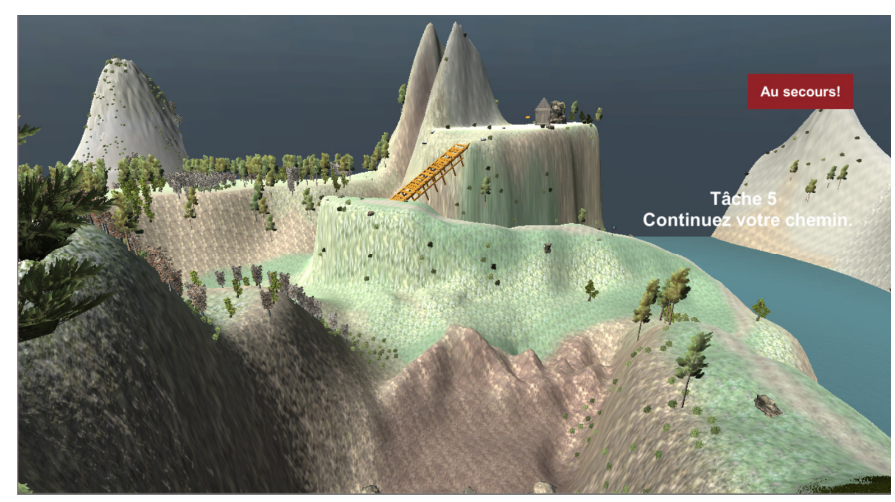

Figure 3: Acrophobia scenario - overview

Iulia-Cristina Stănică, Maria-Iuliana Dascălu, Alin Moldoveanu and Florica Moldoveanu (2017), International Journal of Interactive Worlds, DOI:10.5171/2017. 155350 
Reassuring tasks are also inserted along the way, for instance the search of a rare flower. The most difficult task, the final one, involves the traversal of a steep bridge in order to arrive to the cabin and accomplish the final goal (Fig. 4). This scenario focuses a lot on the little details: the grass, the rocks, the landforms, the wind - all of them are carefully placed in the environment.

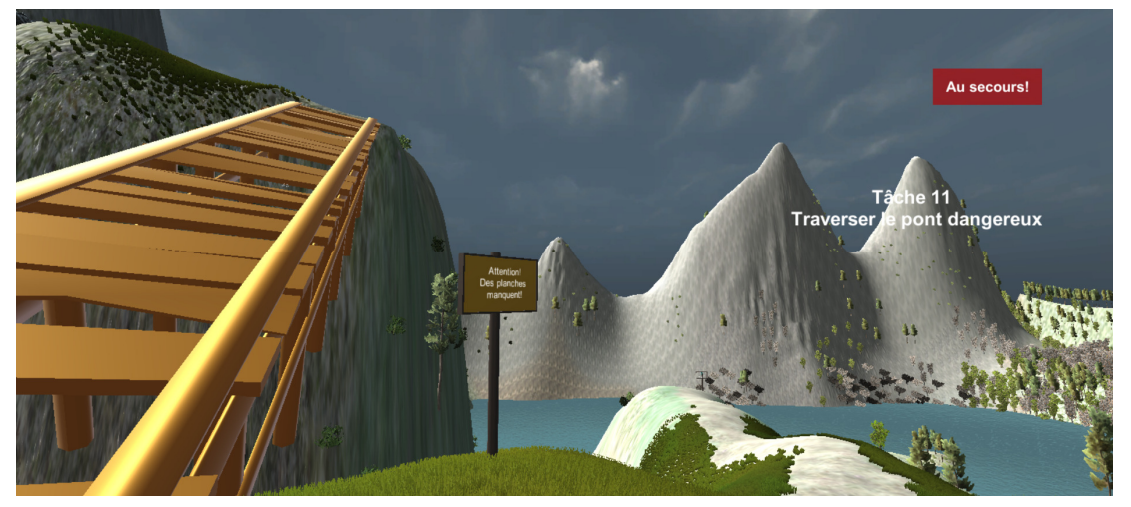

Figure 4: Final task of the acrophobic scenario

\section{Claustrophobia}

As far as claustrophobia goes, the scenario consists in a hospital visit for a medical examination. This module starts with presenting the exterior of the hospital - a city with many streets, buildings and a park, where the user must walk to accustom to the application (Fig. 5). After entering the hospital, the patients must perform several medical visits, at the ground floor or first floor: to the ophthalmologist,

to

the otorhinolaryngology or to prepare for an MRI (magnetic resonance imaging). As the tasks progress, the user encounters narrow halls, full elevators, small and dark rooms. He must maintain his calm until the end, when he enters a MRI machine, and therefore completes the most difficult task. This scenario has many animations, sound effects and lights, to assure the realism and a high level of the sense of presence.

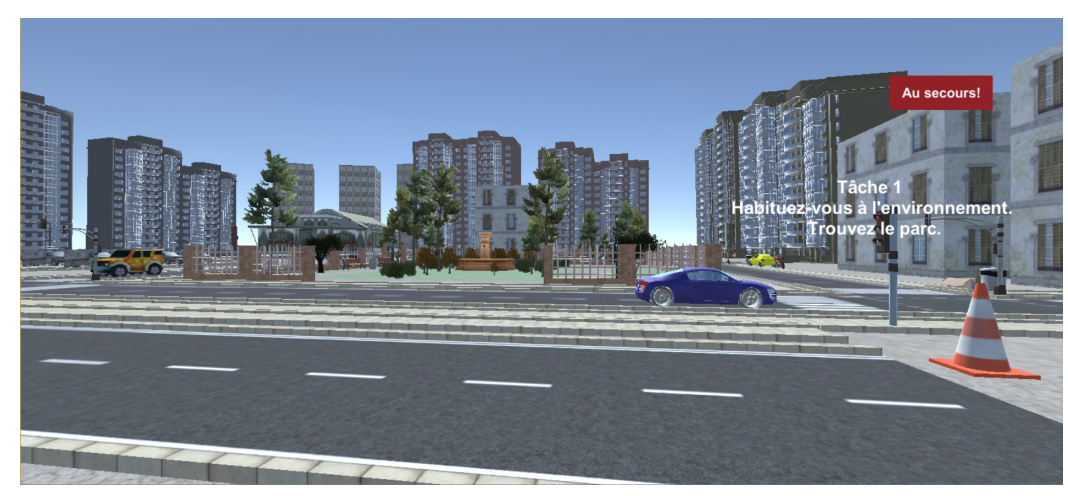

Figure 5: First task of the claustrophobic scenario 


\section{Technical Description}

\section{Architecture}

Even if the application has the goal of creating a realistic environment in order to maximize the immersion and thus focus on the graphics, the programming part is also important for realizing special functionalities, such as animations, interface elements or player controllers.

For the architecture of the Anti-PHOBIES application, we have decided to focus on the one implemented inside the game engine Unity3D: the Entity-Component architecture. This design pattern, the root of the architecture, is widespread in the video game industry. The main idea of this approach is that an entity doesn't have its own logic: it is implemented through its components. An entity is actually made of multiple components, each one having its own, unique goal (Farrell, 2014). This design is extremely logical in this field, where each object of the virtual world is in fact an entity: the player, an enemy, a vehicle or a building. When we want to add a certain behavior to the object, we actually add some components to the entity. It is an easy and flexible approach, mandatory for games (serious or ludic), because these types of applications are created by performing repeated tests, on an accessible and easy to modify code.

\section{Technologies}

Most similar applications which use virtual reality to treat phobias focus on their concept and results, not on the technologies used. Therefore, we had to weigh the existing technical approaches in the game industry in order to choose the better option for our serious game. After analyzing the available options on the market, by comparing their advantages, their drawbacks and taking into consideration the necessities of our application, we have chosen Unity3D as a game engine and SketchUp as a modeling tool. By choosing Unity3D as our game engine, the main technological features are related to its functionalities. The principal aspects of a Unity project are exemplified in Fig. 6, with the characteristics exemplified in our application. We will try to present in a few words each technical aspect of our project.

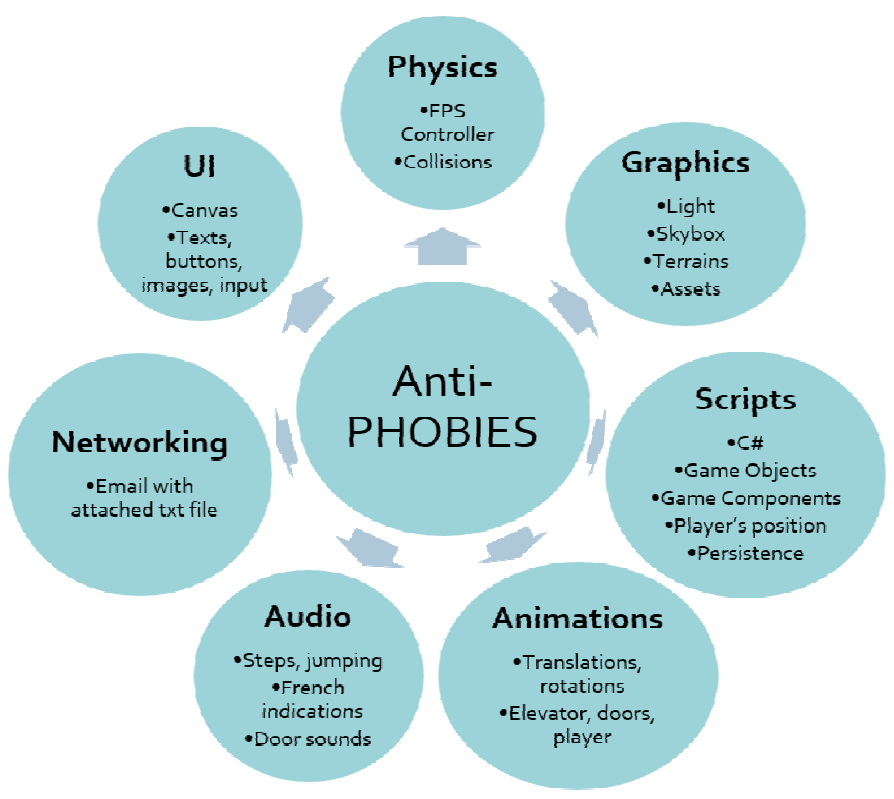

Figure 6: Technical description of the project

Iulia-Cristina Stănică, Maria-Iuliana Dascălu, Alin Moldoveanu and Florica Moldoveanu (2017), International Journal of Interactive Worlds, DOI:10.5171/2017. 155350 
Physics is essential for creating a game, ludic or serious: we should have a plausible acceleration, we should have collisions between the player and the objects of the world, and we should have gravity and other forces to influence the events taking place in the virtual environment.

Graphics are an essential part of our application, since the immersion of the user can be obtained only if the virtual environment is realistic enough to give the impression of being in a real world. Fortunately, Unity3D has countless possibilities of creating spectacular visual effects: lights, materials, textures, shadows etc. It also contains a Terrain Engine, which allows the creation of immense realistic landscapes.

The scripts have the role of managing the player's behavior, controlling other objects, managing the user's input or creating artificial intelligence systems in the game. The programming language that we used for scripting is $\mathrm{C \#}$, an object-oriented language very similar to Java.

Animations are important for improving the realism of a virtual environment. They can be either created inside Unity or in a 3D modeling software and imported afterwards. We have chosen the first option in order to create animations for opening and closing doors, using the elevator or simulating the MRI procedure.

Unity has many functionalities for the audio part - from 3D spatial sounds to realtime mixing. In Unity, the principle is that of transmitter-receiver: an audio source creates the sounds and the audio listener receives them, like a microphone. In our application, the receiver was attached to the camera of the player, while the transmitters were related to various objects in the scene: doors opening or closing, doctor speaking, water flowing.

The user interface is very important, as it represents the first element seen by the user when opening the application. In order to create the main menu and the questionnaire, we used a canvas where we placed the UI elements: image for the background, regular buttons, toggle buttons, user inputs and texts. UI elements can be also present in the scenes of the virtual world, for instance the "help" button which can be pressed by the user in any moment.

Unity offers the opportunity of rendering the application using auxiliary devices, such as head mounted displays. We have tested Anti-PHOBIES using an Oculus Rift and the experience was spectacularly immersive. Future ameliorations can be made, by modifying the camera parameters and using a binocular vision of the project, for a $100 \%$ VR experience.

\section{Validation}

The software validation had two parts: testing the application and having several people fill out a survey. A total number of 39 people from different social environments participated to the validation process: engineers, doctors, employers of the Tehnoplus Industry and Tehnoplus Medical companies, students and teachers from Politehnica University of Bucharest or from the Academy of Economic Studies.

For the testing part, the users were free to use their own computers, after receiving indications related to the ways of using the application. We had thus tested the application's adaptability on totally different platforms. After this step, the test subjects were asked to fill out a questionnaire created using Google Forms and containing four main sections: personal information, questions related to stress, discomfort or sense of presence, technical performance and user interface, as well as the importance of using virtual reality for treating phobias (Balan \& all 2014). It was a balanced distribution of people suffering from claustrophobia, acrophobia, some other phobia or perfectly healthy people, in order to test the application on a great variety of subjects. By using learning analytics approaches, such as content analysis and information visualization, we interpreted the 
questionnaire results to draw some conclusions.

The sense of presence attained high levels for the two phobias $79.7 \%$ and $74.6 \%$ of the respondents considered that the sense of presence was high or very high), showing that the scenes from the application were realistic. The stress level was rather high for the people suffering from a certain phobia and really low for the healthy test subjects. The scenes related to acrophobia seem to cause more stress.
We asked our respondents to give us suggestions in order to ameliorate the sense of presence. We have received really useful propositions: using additional VR devices - because not all of them had the opportunity of testing the application with the Oculus Rift (Fig. 7), - adding more details, modifying the walking speed (making it slower or using several levels of speed), adding animations to the other characters based on the player's actions, adding sensors to detect falls.

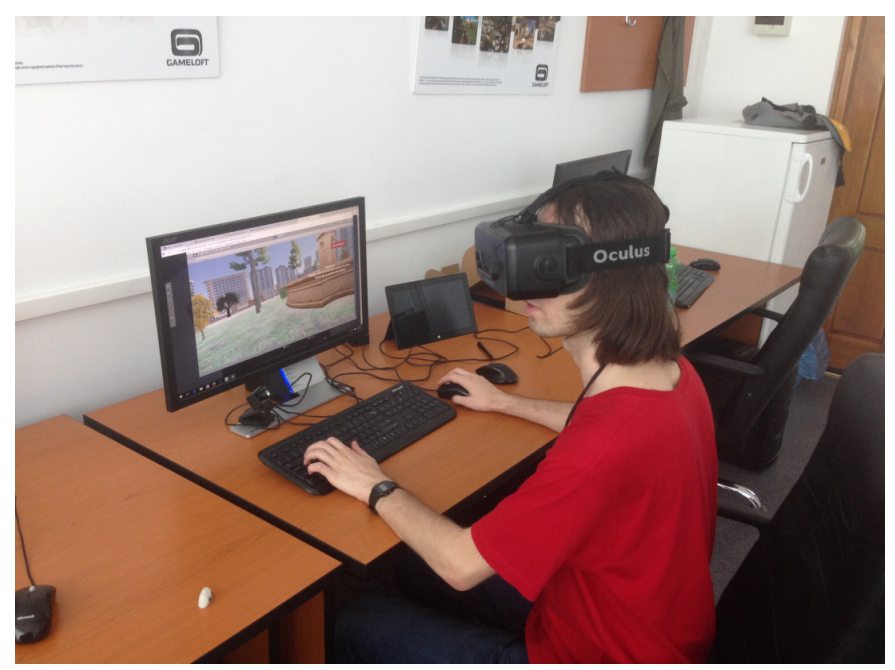

Figure 7: Testing the application with the Oculus Rift

The users were happy about the technical aspects, giving positive feedback to the questions related to the graphics, controls or user interface. The final question, related to the utility of using virtual reality to treat phobias, was well received by the users - they were delighted and open to novelty, giving an average score of 9.49 out of 10 .

In the future, validation will be possible through adding adjacent devices in order to surveil medical parameters - the fitness bracelets like EPOC or the actiCAP devices being some remarkable examples.

\section{Conclusions}

By analyzing specific applications, we can conclude that the use of virtual reality in order to learn how to treat specific phobias can bring satisfying results, taking into consideration the post-treatment effects, reactions or ameliorations felt by the test subjects. The sense of presence, this sensation of being in a certain environment, is an important part of a VR system, which contributes immensely to its success (Juan \& Perez 2010). Real emotions, positive or negative (such as anxiety or fear) are developed during the use of virtual reality applications. All the tests have been conducted on various subjects and the results have been positive, even unbelievable (for instance in the scenario of the Spider-World application and its first patient, Miss M.).

After talking to doctors and psychologists, we have found out that they perceive virtual reality as a great technology, with huge potential, which could be used to 
create various real-life scenarios in order to treat different phobias. There are many advantages of VR applications, such as: great variety of virtual environments, which can be adapted based on the desired phobias, great accessibility and great flexibility to the user's needs or the decreasing of the duration of the therapy. A greater flexibility could also mean an increase of the safety and the control of the therapy, as the "in-vivo" exposure could be unpredictable (Duke Psychiatry and Behavioral Science 2016).

Today, we find ourselves in the age of technology, so it is natural to use it also in the medical field. The significant improvements which were made during the last years can be easily observed in many aspects, such as speed, graphics quality, development of adjacent VR devices or the technology related to the detection of movement. It is why we can observe an increase in the interest shown towards the use of virtual reality to treat mental conditions, especially phobias. In addition, virtual reality is not a recent discovery anymore, which can explain the decrease of development, acquisition, and maintenance costs of a VR system (Turner and Casey 2014).

The "in vivo" therapy can become extremely expensive, as it involves travelling to a certain destination where the stimuli desired for the patient's phobia can be provoked. Sessions are therefore extended. In the case of virtual reality, the same exposure to the stimuli can be done in a standard therapy. The patient does not have to leave the therapist's office, so the costs are reduced considerably. The problem of insurance companies that do not want to pay for extended treatment sessions is also solved: VR based sessions have a short duration of 45-50 minutes (Duke Psychiatry and Behavioral Science 2016).

Our paper did an analysis of the current methods used for treating phobias, especially virtual reality. The goal of our project was to create a virtual reality application to treat phobias, more specifically claustrophobia and acrophobia.
By using the Unity3D game engine, we had successfully created an interactive virtual environment, which could be very useful as a means of treatment. The software can be adapted anytime for mobile devices or for a better use on virtual reality devices (Oculus Rift or Google cardboard). The flexible architecture of the application will allow us to add more modules, to treat a larger range of phobias. A future version of the application can also add a module for providing therapy under the surveillance of the therapist. The doctor will have the possibility of controlling dynamically the changing of the environment. A module for analyzing the progress of therapy could also be implemented, based on the doctor's remarks.

If we analyze the advantages of virtual reality, we can observe it is a great technology, which evolves continuously and which has to be constantly improved. Virtual reality is a useful tool to treat phobias, but there are also other conditions which could be solved with the help of this technology. We have many opportunities, we just have to keep trying to find solutions and achieve the ultimate goal improving the quality of people's lives.

\section{Acknowledgment}

This work has been funded by University Politehnica of Bucharest, through the "Excellence Research Grants" Program, UPB - GEX. Identifier: UPB-EXCELENȚĂ2016 "Construirea si exploatarea profilului utilizatorilor in medii de invatare virtuale: metode, implicatii psihosociale si consecinte utilizand tehnici de analiza avansata a datelor - LeProVE (Learning Profiling in Virtual Environments)", Contract number 83/26.09.2016, code 303 . (LeProVE). The work has also received funding from the European Union's Horizon 2020 research and innovation program under grant agreement No 643636 "Sound of Vision" and from the TRAVEE grant of the Romanian Executive Agency for Higher Education, Research, Development and Innovation Funding UEFISCDI, Joint Applied Research Projects program, project number PN-II-PT-PCCA2013-4-1580. 


\section{References}

1. Adam, M., (2013), Entity Systems are the Future of MMOG Development, T-Machine

2. Alonso, J., Angermeyer, M. C., Bernert, S., Bruffaerts, R., Brugha, T. S. and Bryson, H., (2004), Prevalence of mental disorders in Europe: results from the European study of the epidemiology

3.Arlington, (2003), Diagnostic and Statistical Manual of Mental Disorders (DSM), American Psychiatric Association

4. Aslandere, T., Dreyer, D., Pankratz, F., Schubiotz, R., A Generic Virtual Reality Flight Simulator

5. Bălan, O., Moldoveanu, A., Moldoveanu, F. and Dascălu, M.-I.,(2014), Navigational 3D audio-based game-training towards rich auditory spatial representation of the environment, System Theory, Control and Computing (ICSTCC), 18th International Conference

6. Chan. J. C.P., Leung, H., Tang, J. K.T., Komura, T., (2011), A Virtual Reality Dance Training System Using Motion Capture Technology, IEEE transactions on learning technologies, VOL. 4, NO.2

7. Coelho, C.M., Waters, A. M., Hine, T.J. and Wallis, G., (2009), The use of virtual reality in acrophobia research and treatment, School of Human Movement Studies, University of Queensland

8. Counseling Directory, Cognitive behavioral therapy, [Online], www.counselling-directory.org.uk

9. Dascalu, M-I., Moldoveanu and A.,Shudayfat, E.A., (2014), Mixed Reality to Support New Learning Pradigms, System Theory, Control and Computing (ICSTCC), 18th International Conference

10. Duke Psychiatry and Behavioral Science, Duke University School of Medicine, [Online], http://psychiatry.duke.edu/
11. Farell, S., (2014), A Critique of the entity component model, GameDev

12. Gamberini, L., Chittaro, L., Spagnolli, A. and Carlesso, C., (2015), Psychological response to an emergency in virtual reality: Effects of victim ethnicity and emergency type on helping behavior and navigation

13. Hoffman, H.G., (2014), Virtual reality therapy, Scientific American

14. Hoffman,H.G., Garcia-Palacios, A., Carlin, C., Furness, T.A. and Botella-Arbona, C.,(2003),Interfaces that heal: Coupling real and virtual objects to cure spider phobia, International Journal of Human-Computer Interaction, 283-300

15. Insel, T., (2011), The global cost of mental illness

16. Joseph, J.S. and Gray, M.J., (2008), Exposure Therapy for Posttraumatic Stress Disorder, Journal of Behavior Analysis of Offender and Victim: Treatment and Prevention

17. Juan, M.C. and Perez, D.,(2010), Using augmented and virtual reality for the development of acrophobic scenarios. Comparison of the levels of presence and anxiety, Universidad Politecnica de Valencia

18. Matthews, A., Naran, N. and Kirkby, K.C., Symbolic online exposure for spider fear: Habituation of fear, disgust and physiological arousal and predictors of symptom improvement, Division of Psychology, School of Medicine, University of Tasmania, Australia

19. Moglia, A., Ferrari, V., Ferrari, M., Mosca, F., Cuschieri, A., (2016), A systematic review of virtual reality simulators for robot-assisted surgery, European Urology 69 1065-1080

20. Moldoveanu, A., (2008), Highlyscalable server for massive multi-player 3D virtual spaces based on multi-processor graphics cards, Annals of DAAAM for 2008 \& Proceedings of the 19th International DAAAM Symposium

Iulia-Cristina Stănică, Maria-Iuliana Dascălu, Alin Moldoveanu and Florica Moldoveanu (2017), International Journal of Interactive Worlds, DOI:10.5171/2017. 155350 
21. PHOBOS application user guide, (2014), Psychological Technologies

22. Rammirez-Moreno, D., (2012), A computational model for the modulation of the prepulse inhibition of the acoustic startle reflex, Biological Cybernetics, 169

23. Smith, M., Segal, R. and Segal, J.: Phobias and Fears, Symptoms, Treatment, and Self-Help for Phobias and Fears, Helpbook

24. Soeter, M. and Kindt, M., An Abrupt Transformation of Phobic Behavior after a Post-Retrieval Amnesic Agent

25. Stanica, I-C., Dascalu, M-I., Moldoveanu, A., Bodea, C-N. and Hostiuc, S.,(2016), A survey of virtual reality applications as psychotherapeutic tools to treat phobias, The 12th International Scientific Conference eLearning and Software for Education (eLSE), Bucarest
26. Turner, W. A. and Casey, L.M., Outcomes associated with virtual reality in psychological interventions: where are we now, (2014), Clinical Psychology Review, 634-644

27. Wittchen,H.U., Jacobi, F., Rehm, J., Gustavsson, A., Svensson, M., Jönsson, B., Olesen, J., Allgulander, C., Alonso, J., Faravelli, C., Fratiglioni, L., Jennum, P., Lieb, R., Maercker, A., van Os, J., Preisig, M., Salvador-Carulla, L., Simon, R. and Steinhausen, H.-C., (2011), The size and burden of mental disorders and other disorders of the brain in Europe 2010, ECBN/EBC REPORT

28. Wolitzky-Taylor, K.B., Horowitz, J.D., Powers, M.B. andTelch, M.J., (2008), Psychological approaches in the treatment of specific phobias: A meta-analysis, , Laboratory for the Study of Anxiety Disorders, Department of Psychology, The University of Texas at Austin, United States 